\title{
La scapula alata dynamique : un diagnostic à évoquer en cas de douleur scapulaire
}

\section{Primary scapular winging: a diagnosis to evoke in case of scapular pain}

\author{
K. Shiraishi $\cdot$ J. Navarro $\cdot$ S. Mayer \\ Reçu le 30 décembre 2017; accepté le 4 avril 2018 \\ (C) SFMU et Lavoisier SAS 2018
}

Un patient de 32 ans, a été admis aux urgences pour une scapulalgie droite évoluant depuis 24 heures, sans notion de traumatisme. L'examen clinique au repos était sans particularité (Fig. 1A), l'appui des membres supérieurs contre un mur provoquait une saillie postérieure de la scapula droite (Fig. 1B). Le bilan biologique était normal. Le médecin urgentiste a évoqué le diagnostic de scapula alata dynamique (SAD). L'électromyogramme (EMG) réalisé par la suite a confirmé une souffrance mono tronculaire du nerf thoracique long (NTL) à droite. Les sérologies virales et Lyme sont revenues négatives. La scapula alata est une pathologie rare. C'est une sous-entité des dyskinésies scapulaires [1]. Elle est dite «dynamique » lorsque le décollement varie avec les mouvements du membre supérieur. Elle résulte d'une insuffisance de fixation de la scapula sur le thorax, secondaire à une atteinte neuromusculaire. Elle est provoquée par l'atteinte du NTL qui innerve le muscle dentelé antérieur ou plus rarement du nerf accessoire qui innerve le muscle trapèze. Elle est principalement d'origine traumatique, inflammatoire ou idiopathique. Le tableau clinique d'une SAD par atteinte du NTL associe une scapulalgie, un décollement de la scapula lors de l'appui contre un mur (Test de wall pushup qui recherche un déficit isolé du dentelé antérieur) [1], un éloignement de l'angle inférieur de la scapula par rapport à la ligne des épineuses au repos et parfois une impossibilité d'élever le bras au-dessus de $90^{\circ}$. Un EMG réalisé sans urgence permet de déterminer les muscles concernés et leurs

\footnotetext{
K. Shiraishi $(\bowtie) \cdot J$. Navarro

Service des urgences, centre hospitalier Jura Sud,

55, rue du Docteur-Jean-Michel - CS 50364,

F-39016 Lons-le-Saunier cedex, France

e-mail : kenji.shiraishi@hopitaux-jura.fr

\section{S. Mayer}

Service de neurologie, centre hospitalier Jura Sud,

55, rue du Docteur-Jean-Michel - CS 50364,

F-39016 Lons-le-Saunier cedex, France
}
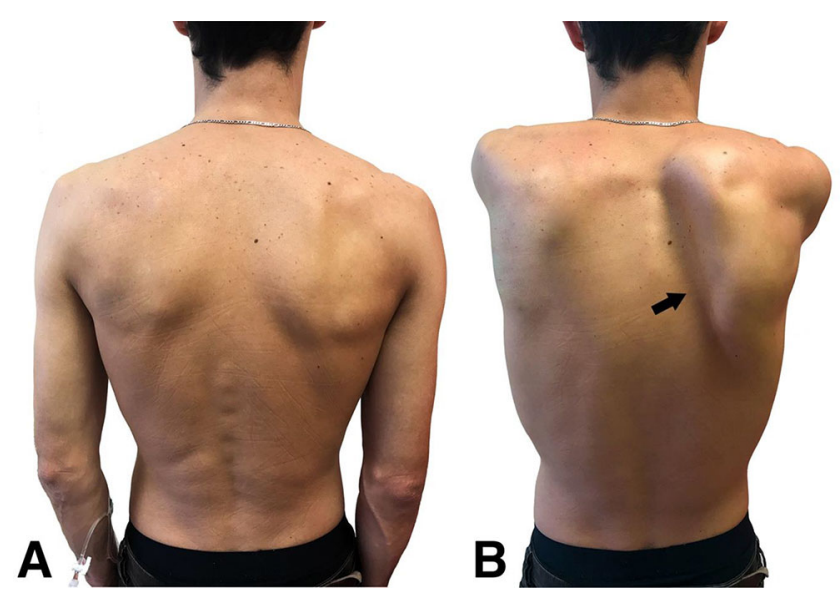

Fig. 1 A. Patient au repos. B. Scapula alata dynamique majorée lors du test wall push-up. Flèche : saillie du bord spinal de la scapula, proche du rachis dorsal

degrés de dénervation. L'IRM de la région scapulaire recherche les signes de dénervation musculaire [2]. Il n'est pas obligatoire mais peut écarter des diagnostics différentiels (compression nerveuse, lésion tumorale). Le pronostic est souvent favorable. Le traitement repose sur une rééducation sollicitant les muscles synergiques du dentelé antérieur en conservant la souplesse musculo-tendineuse autour de la scapulo-humérale. En conclusion, une scapulalgie doit toujours être examinée de manière dynamique. Le test de wall push-up est un test simple pour mettre en évidence une SAD. La prise en charge est multidisciplinaire.

\section{Références}

1. Nguyen C, Guérini H, Roren A, et al (2015) Neuromuscular dynamic scapular winging: Clinical, electromyographic and magnetic resonance imaging diagnosis. Presse Med 44:1256-65

2. Friedenberg SM, Zimprich T, Harper CM (2002) The natural history of long thoracic and spinal accessory neuropathies. Muscle Nerve 25:535-9 